\title{
Barriers and facilitators associated with colonoscopy completion in individuals with multiple chronic conditions: a qualitative study
}

\author{
Shahnaz Sultan ${ }^{1-4}$ \\ Melissa R Partin ${ }^{1,2}$ \\ Phalgoon Shah ${ }^{5}$ \\ Jennifer LeLaurin ${ }^{4}$ \\ Ivette Magaly Freytes ${ }^{4}$ \\ Chandylen L Nightingale ${ }^{6}$ \\ Susan F Fesperman ${ }^{4}$ \\ Barbara A Curbow ${ }^{7}$ \\ Rebecca J Beyth ${ }^{3,4,8}$ \\ 'Center for Chronic Disease \\ Outcomes Research, Minneapolis \\ Veterans Affairs Health Care System, \\ ${ }^{2}$ Department of Medicine, University \\ of Minnesota, Minneapolis, MN, \\ ${ }^{3}$ Department of Medicine, University \\ of Florida College of Medicine, \\ Gainesville, FL, ${ }^{4}$ Center of Innovation \\ on Disability and Rehabilitation \\ Research, North Florida/South \\ Georgia Veterans Health System, \\ Gainesville, FL, ${ }^{5}$ Department of \\ Medicine, Tripler Army Medical \\ Center, Honolulu, HI, ${ }^{6}$ Department \\ of Social Sciences and Health Policy, \\ Wake Forest School of Medicine, \\ Winston-Salem NC, ${ }^{7}$ Department of \\ Community and Behavioral Health, \\ University of Maryland, College Park, \\ MD, ${ }^{8}$ Geriatric Research, Education \\ and Clinical Center, North Florida/ \\ South Georgia Veterans Health \\ System, Gainesville, FL, USA
}

Correspondence: Shahnaz Sultan Center for Chronic Disease Outcomes Research, Minneapolis Veterans Affairs Health Care System, One Veteran Drive, Minneapolis, MN 554I7, USA

$\mathrm{Tel}+\mathrm{I} 6124674364$

Fax + I 6127175699

Email shahnaz.sultan@va.gov
This article was published in the following Dove Press journal:

Patient Preference and Adherence

24 May 2017

Number of times this article has been viewed

\begin{abstract}
Background: A recommendation to undergo a colonoscopy, an invasive procedure that requires commitment and motivation, planning (scheduling and finding a driver) and preparation (diet restriction and laxative consumption), may be uniquely challenging for individuals with multiple chronic conditions (MCCs). This qualitative study aimed to describe the barriers and facilitators to colonoscopy experienced by such patients.
\end{abstract}

Materials and methods: Semistructured focus groups were conducted with male Veterans who were scheduled for outpatient colonoscopy and either failed to complete the procedure or completed the examination. Focus group recordings were transcribed and analyzed by an inductive grounded approach using constant comparative analysis.

Results: Forty-four individuals aged 51-83 years participated in this study (23 adherent and 21 nonadherent). Participants had an average of 7.4 chronic conditions (range 2-14). The five most common chronic conditions were hypertension (75\%), hyperlipidemia (75\%), osteoarthritis/ degenerative joint disease (59\%), atherosclerotic heart disease (48\%), and diabetes mellitus $(36 \%)$. We identified four unique themes that influenced motivation to undergo a colonoscopy: competing medical priorities, low perceived benefit, a prior negative colonoscopy experience, and pre-existing medical conditions. Additionally, we identified four themes that influenced individuals' ability to complete the examination: difficulty with bowel cleansing, difficulty with travel, worry about exacerbation of pre-existing conditions, and heightened concerns about potential complications.

Conclusion: MCCs are common in individuals referred for colonoscopy and generate unique barriers to colonoscopy completion related to medication, dietary changes, transportation, preparation processes, symptoms exacerbation, and complication concerns. Future research should examine whether tailored interventions that include education and support in addressing the unique barriers can enhance colonoscopy completion.

Keywords: adherence, colonoscopy barriers, multiple chronic conditions, Veterans

\section{Introduction}

Over the past decade, efforts to promote patient-centered care for individuals with chronic conditions have included moving away from disease-specific approaches to strategies that consider patients' personal goals, their social context, and their ability to self-manage. ${ }^{1,2}$ Chronic conditions are defined as conditions lasting 12 months or more that limit self-care and independent living and require ongoing medical intervention. ${ }^{3-5}$ According to recent estimates, approximately half of adults in the US have at least one chronic condition and more than one in four Americans have multiple chronic conditions (MCCs). ${ }^{5-7}$ Individuals with MCCs have high rates of health care utilization 
and spending. ${ }^{8,9}$ With the aging of the US population, the number of Americans with MCCs is projected to be 81 million by $2020 .{ }^{10}$ Certain subpopulations, such as Veterans and older adults, have especially high rates of comorbidities. ${ }^{9,11,12}$

Understanding how chronic conditions impact patients and their ability to adhere to health care recommendations is critical to provide more patient-centered care. ${ }^{12,13}$ Adherence, in this group of individuals, may be influenced by the overall burden of existing medical conditions, knowledge and understanding of the condition, capacity for self-management, as well as treatment complexity and feasibility. ${ }^{14}$ For example, a recommendation to undergo a colonoscopy, an invasive procedure that requires motivation, planning (scheduling and finding a driver), and preparation (diet restriction and laxative consumption), may be uniquely challenging for individuals with MCCs as a result of varying levels of illness severity and disability. ${ }^{12}$ Many published studies have explored barriers to colonoscopy, but there is limited information on the challenges of undergoing colonoscopy in the context of having MCCs. Prior studies focusing on chronic conditions have primarily examined the association between colonoscopy utilization and the number and types of comorbid conditions using large administrative datasets. ${ }^{15,16}$ However, an in-depth patient perspective is markedly missing from the published literature.

To fill this gap in the literature, we sought to characterize the range of barriers to colonoscopy completion experienced by patients with MCCs using a qualitative approach. Qualitative studies of patients' perspectives on undergoing colonoscopy in the setting of MCCs are essential for the design of effective interventions in this population.

\section{Materials and methods Design}

We conducted a qualitative study using focus groups to obtain in-depth information about potential barriers associated with colonoscopy completion from the patients' perspective. We focused on individuals who had been referred for colonoscopy by their provider and either successfully completed the procedure or were nonadherent to their colonoscopy appointment, despite intending to complete the examination. The institutional review board of the University of Florida and the Research and Development Committee of the Malcom Randall Veterans Affairs Medical Center (VAMC) approved the study. Reporting of this study adheres to the COREQ requirements. ${ }^{17}$

\section{Participants and procedure}

We identified potential participants by searching lists of consecutive patients referred and scheduled for colonoscopy from May 2009 to May 2010 at the Malcom Randall VAMC in Gainesville, FL, USA. Eligible participants included English-speaking men between the ages of 50 and 85 years with an established primary care provider at the North Florida/South Georgia Veterans Affairs Health System (NF/SGVHS) who had been referred for a colonoscopy for the following indications: surveillance, diagnostic evaluation of symptoms or a positive fecal occult blood test, or highrisk screening due to a positive family history. Participants were 50-85 years because current guidelines recommend colorectal cancer screening for all individuals starting at age 50 years until age 85 years (depending on overall health and screening history). ${ }^{18}$ We classified individuals as adherent if their electronic health record indicated that they had completed the colonoscopy within 12 months and nonadherent if they had failed to do so in the 12-month period. Some patients who were considered nonadherent had completed a prior colonoscopy but were nonadherent for their most recent visit. Additionally, we recruited individuals from rural and urban areas of residence to ensure that we captured any barriers related to geographic distance or travel time to the medical center.

We mailed opt out letters to potential participants, and then telephoned those who did not opt out to invite them to participate. We maintained a rolling recruitment until at least 8 to 10 Veterans verbally agreed to participate in each focus group. We completed seven focus groups consisting of six to eight participants each; four consisted of adherent Veterans and three consisted of nonadherent Veterans. Of the 600 eligible individuals identified, 267 could not be contacted, 256 declined, and 77 agreed to participate. Ultimately, 44 Veterans attended the focus groups (Figure 1). Participants gave written informed consent and received a \$20 gift card.

\section{Data collection}

We had two focus group facilitators with prior training and experience in conducting qualitative studies. One expert in focus group methodology (BC) facilitated four groups and the lead investigator (SS) facilitated the other three groups. We used a semistructured interview guide based on prior published barriers to colonoscopy to facilitate the group discussions (Table 1). Focus groups started with open-ended questions regarding patients' experiences with colonoscopy followed by more detailed and probing questions. Each focus group lasted 60-90 minutes, and refreshments were provided. Following completion of the session, each participant completed a short questionnaire that included sociodemographic questions. All focus groups were audiorecorded and transcribed verbatim. 


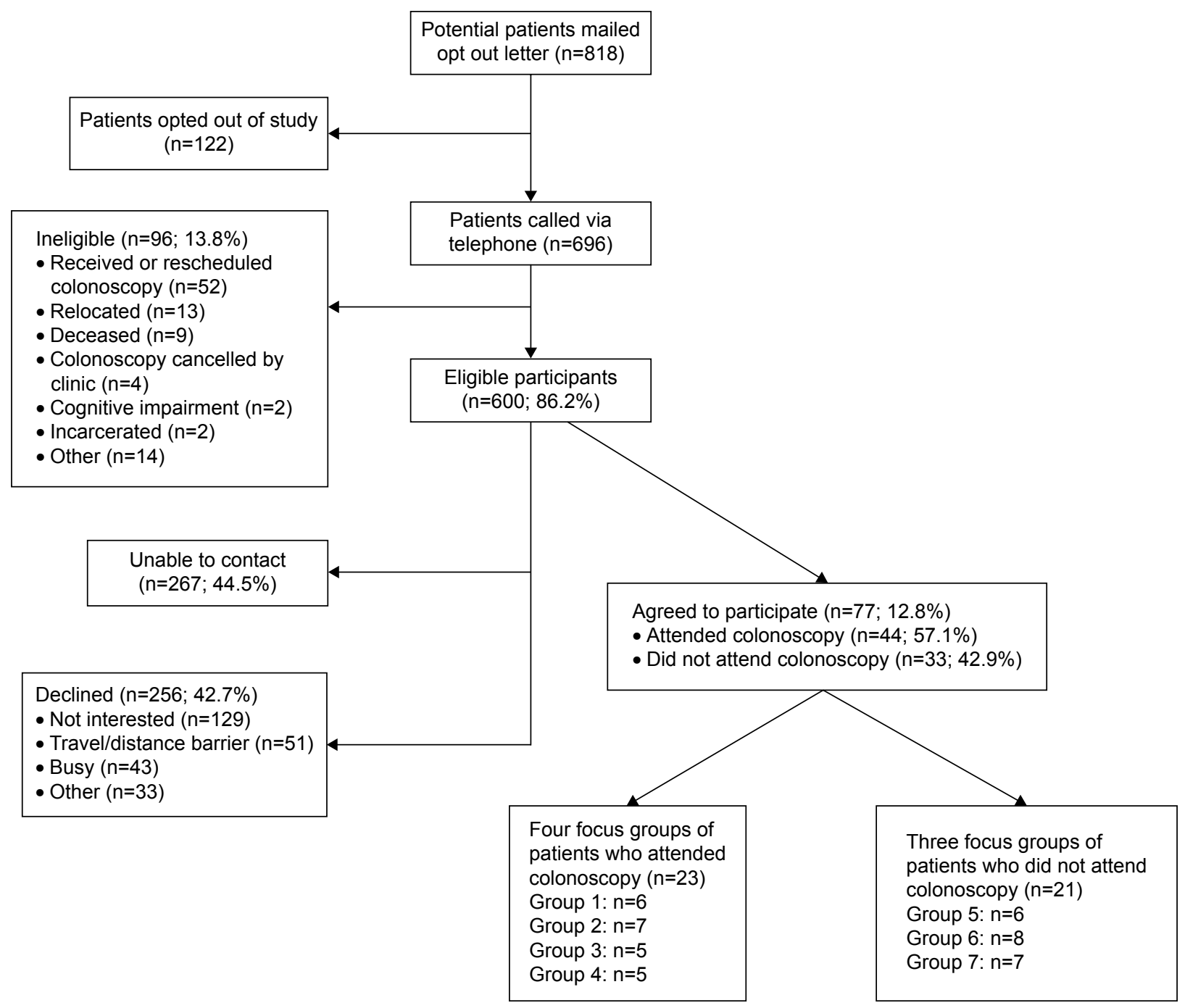

Figure I Participant recruitment flowchart.

\section{Analysis}

An inductive grounded approach was used for the qualitative analysis of the transcripts using constant comparative analysis, allowing for themes to emerge from the collected data and progress through three defined processes: description, categorical/conceptual ordering, and theorizing. ${ }^{19,20}$ This involved an iterative process of reading and re-reading transcripts, developing a coding scheme to reflect unique ideas, applying the coding scheme to transcript text, and grouping coded text by theme. We used both open and axial coding. Analyzing transcripts line-by-line in their entirety, we used open coding to identify ideas or concepts mentioned by study participants and then grouped concepts together to form categories and subcategories. We used axial coding to make connections between categories and subcategories. We collapsed and grouped codes generated from open coding into mutually exclusive categories focusing on three interrelated aspects of Straus and Corbin's coding paradigm: individual actions or behaviors, situational context, and consequences of behaviors. ${ }^{20} \mathrm{We}$ assembled recurrent ideas into themes based on content similarity. We reviewed and assembled these themes into theoretical constructs based on their relation to one another and their ability to explain factors influencing colonoscopy completion. Themes and theoretical constructs were tabulated to compare barriers to facilitators. To minimize the chance that important thematic ideas were overlooked, the research team independently analyzed and coded transcripts. Research team members involved in the analysis included a gastroenterologist/ health services researcher (SS), a geriatrics health services researcher (RB), a qualitative methods expert (IMF), and a research assistant with qualitative experience (JL). Two investigators (SS and RB) began by independently examining and assigning descriptive codes to segments of transcripts. Two additional team members (IMF and JL) discussed and reviewed these codes and added codes as they reviewed 
Table I Focus group guiding questions

Who suggested that you should have a colonoscopy?

How was it communicated?

What were some factors that made it difficult to undergo a

colonoscopy?

Were there any specific hurdles that made it hard for you to attend

your appointment?

For those who had a colonoscopy in the past:

What was the experience like for you?

Did any of the following factors make it harder or easier to undergo a

colonoscopy?

Access, cost, embarrassment, bowel preparation, distance, concern

for pain, and worry

subsequent transcripts. Coders noted differences in codes and themes across focus groups and reconciled differences in coding across coders by discussion until consensus was reached, and the codes were modified accordingly. This ensured that the organization of the data and the resultant conceptual theory was transparent. From this primary analysis of the data (Table S1), we pursued a more intensive focus on identifying barriers among individuals with chronic conditions. We went back to the original transcriptions and reviewed the findings to select representative quotes from the transcripts to illustrate the final themes. Although we did not include the presence of multiple comorbidities as eligibility criteria for the initial study, we used medical record data to identify the number and type of chronic conditions among participants. We used NVIVO 8 (QSR International) to manage our data and SPSS 21.0 (IBM Corporation) to perform descriptive statistics to summarize demographic data. Because the themes emerging from the groups limited to individuals who had completed a colonoscopy were similar to those emerging from the groups limited to individuals who did not complete a colonoscopy, we present results for both groups together.

We compiled data on comorbid or chronic conditions after focus group completion and transcription using the problem list in the electronic health record. Comorbidity was defined using the Charlson-Deyo Comorbidity Index, which is a summary of 17 chronic disease diagnoses from administrative data selected and weighted according to their association with mortality. ${ }^{21,22}$ Based on prior studies, we categorized patients as having no significant morbidity if they had a Charlson score $=0$, average comorbidity if they had a Charlson score $=1-3$, and severe comorbidity if they had a Charlson score $>4 .{ }^{16,23}$ Additionally, we summarized data on the total number and types of chronic conditions.

\section{Results}

A total of 44 Veterans participated in the focus group discussions (Figure 1). Demographic and health characteristics of the participants are shown in Table 2 (note: one patient did not complete the questionnaire). The age of the participants ranged from 51 to 83 years (mean 66.2 years). The majority were white $(83.7 \%)$ and married or cohabitating $(62.8 \%)$. Twenty-one percent of participants had less than or equivalent to a high school education, while $32.6 \%$ had some college education. Approximately $40 \%$ of the participants lived in rural areas. Twenty-one patients $(47.7 \%)$ had been nonadherent to their colonoscopy appointment, failing to complete the examination. The mean number of chronic conditions was 7.4 with a range of $2-14$. Approximately $23 \%$ of participants had over 10 chronic conditions. The five most common conditions were hypertension (75\%), hyperlipidemia $(75 \%)$, osteoarthritis/degenerative joint disease (59\%), atherosclerotic heart disease (48\%), and diabetes mellitus (36\%). Based on the Charlson-Deyo Comorbidity Index, a validated tool

Table 2 Demographic and health characteristics of participants $(\mathrm{N}=44)$

\begin{tabular}{ll}
\hline Characteristics & $\mathbf{N}(\%)$ \\
\hline Age, mean years (SD), range & $66.2(7.9), 51-83$ \\
Race & \\
White/Caucasian & $36(83.7)$ \\
Black/African American & $5(11.6)$ \\
Other/Hispanic & $2(4.7)$ \\
Marital status & \\
Married/cohabitating & $27(62.8)$ \\
Separated/divorced/widowed & $16(37.2)$ \\
Education & \\
$\leq$ High school & $9(20.9)$ \\
Some college & $14(32.6)$ \\
$\geq$ College degree & $20(46.5)$ \\
Adherence to colonoscopy appointment & \\
Adherent & $23(52.3)$ \\
Nonadherent & $21(47.7)$ \\
Locality & \\
Rural & $18(40.2)$ \\
Nonrural & $26(59.1)$ \\
Charlson-Deyo Comorbidity Index & \\
0 (none) & $9(20.4)$ \\
I-3 (average comorbidity) & $17(38.6)$ \\
$>4$ (severe comorbidity) & $18(40.9)$ \\
Number of chronic conditions & \\
Mean number, range & $7.4,2-14$ \\
$0-3$ & $5(11.4)$ \\
$4-6$ & $15(34.1)$ \\
$7-10$ & $14(31.8)$ \\
$>10$ & $10(22.7)$ \\
\hline Note:Mssing &
\end{tabular}

Note: Missing data for one participant. 
that weighs comorbidity factors such as cardiovascular disease, dementia, chronic obstructive pulmonary disease, and rheumatologic disease, scores ranged from 0 to $16 ; 20.4 \%$ of participants $(n=9)$ had a Charlson score $=0$ (none) and $40.9 \%$ had a Charlson score $>4$ (severe comorbidity).

\section{Themes}

We identified a number of barriers and facilitators associated with colonoscopy completion. The full coding tree is included in Table S1. The "Results" and "Discussion" sections focus on the perceived barriers to colonoscopy completion in the context of having MCCs (Table 3).

We describe four themes that influenced motivation to undergo a colonoscopy: 1) competing medical priorities, 2) low perceived benefit, 3) a prior negative colonoscopy experience, and 4) having pre-existing medical conditions. We also identified four themes that influenced their ability to complete the examination: 1) difficulty with bowel cleansing, 2) worry about exacerbation of pre-existing conditions, 3) difficulty with travel, and 4) heightened concerns about potential complications.

Themes related to how chronic conditions influence motivation to complete colonoscopy.

\section{I) Competing medical priorities}

Competing medical priorities was emphasized as an important barrier to completing colonoscopy. Individuals talked about how they had to delay their colonoscopy or schedule their colonoscopy around the routine medical management

Table 3 Themes identified in individuals with chronic conditions

Themes related to how chronic conditions influence motivation to complete colonoscopy

I. Competing medical priorities affecting decision to complete colonoscopy

2. Low perceived benefit from colonoscopy because of chronic conditions

3. Prior negative colonoscopy experience in the context of chronic conditions

4. Pre-existing medical conditions as a motivator for undergoing colonoscopy

Themes related to how chronic conditions influence ability to complete colonoscopy

I. Difficulty tolerating the laxative for bowel cleansing

2. Difficulty with travel because of chronic conditions

3. Exacerbation of pre-existing conditions due to the changes in medications and diet

4. Heightened concerns about safety and potential complications because of chronic conditions of their other chronic conditions. One participant described this by saying:

I want to get to where I can see well and I have a heart condition that I'm dealing with too. I understand colonoscopies are very important and I do want to get a clear bill of health, but these other issues are a little more pressing.

Another participant said:

I had to put it off for a while because I had been dealing with a detached retina. I probably made 30 trips at least this past year, so I had to work it in between surgeries.

And a third participant stated:

Mine was delayed a year because I had surgery last year, so they didn't want to do anything.

\section{2) Low perceived benefit}

Some individuals deemed that colonoscopy would be of less benefit to them because their pre-existing medical conditions have a bigger impact on their overall health. Their motivation to complete the colonoscopy was influenced by this idea of a low perceived benefit. One participant said:

I've got some other serious health issues. Let's deal with what I know I've got, rather than what I might have.

Another participant discussed how his chronic conditions were going to impact his overall life expectancy more than a colonoscopy would:

I've got 4 or 5 other things that're going to kill me way before anything going on with my colon.

\section{3) Prior negative experience}

Another important barrier that emerged among individuals who had previously completed a colonoscopy was a prior negative experience. In the context of their chronic conditions, these negative experiences included: ineffective sedation during the prior colonoscopy (primarily because of cardiorespiratory issues) and increased postprocedure side effects that were exacerbated by their pre-existing conditions. One participant noted that because of his other cardiorespiratory problems, they were unable to give him enough sedation:

They could never knock me out. I was awake [...] but my respiratory, my heart beats very low $[\ldots]$ so they were afraid to knock me out. 
Another participant who had diabetes described how bad he felt after the colonoscopy and said:

You go two days without eating anything and they get you cleaned out and then you're starving to death [...] but when it's over you're so sick and nauseous, and being a diabetic, you have so much sugar in you.

\section{4) Having pre-existing medical conditions}

Although the themes described previously served as deterrents to undergoing a colonoscopy and were predominately shared by nonadherent Veterans, adherent participants emphasized that the presence of comorbid pre-existing conditions actually served as a motivator to complete the colonoscopy. A few participants talked about the value of diagnosing a condition that could then be treated. One participant said:

I think it's important [...] I had a blood test 4 years ago and I'm glad I had it [...] I have leukemia and I take chemo every day. So had I not had that test, I may not be here, and I feel the same way about the colonoscopy.

Another participant said:

I kept every now and then urinating a little drop of blood [...] so I went and they find out that I also had high blood (pressure) and a couple of kidney stones [...] see at your age it can be cancer and I know some of them that had cancer, and they died a pretty miserable death. So I said that I'm willing to let them go and find out.

Additionally, participants expressed how knowing about a medical problem was important because it then allowed for appropriate management. For example, one participant said:

I have diabetes, hypertension, I can live with that. I can control it. But when I don't know what's wrong, I can't control it. That motivated me [...].

Themes related to how chronic conditions influence ability to complete colonoscopy.

\section{I) Difficulty with bowel cleansing}

Some individuals with chronic medical conditions were concerned that their medical conditions might impede their ability to drink the large volume of laxative that was needed for bowel cleansing (difficulty tolerating the laxative because of chronic conditions). For some of these individuals, drinking the large jug of laxative over a short period of time exacerbated their symptoms of nausea and contributed to bloating or abdominal discomfort. The quotes from two different participants are as follows:

I can drink a half a gallon and I'm so bloated [...] I'm a diabetic, if it's my diabetes has anything to do with it, I'm on an insulin pump.

$[\ldots]$ when I combine it with my diabetes. I have kidney problems [...] and this Golytely, it bloats me up for some reason and I get it down and I'll be sitting and watching TV and I mess myself up and I'm still having problems with it.

\section{2) Worry about exacerbation of pre- existing conditions}

Some individuals expressed concern that the medication adjustments and dietary changes would exacerbate their underlying chronic conditions. This was especially a concern for individuals with issues related to glycemic control. One participant said:

I'm diabetic and the day of colonoscopy you cannot take your diabetic medicine and so you're already thinking what do you do [...] I have to eat before I put insulin in myself otherwise I'll pass out cause I'm low [...] It's even more harder if you're a diabetic with this.

Another participant expressed his concern about stopping his arthritis medication and how this might impact his body:

I have some arthritis [...] they told me to stop the arthritic medication 5 days prior [...] sitting still such a long time and going to recovery and laying there for a while, oh God, this is going to be tough.

\section{3) Difficulty with travel}

A few individuals expressed concern over their ability to travel to the endoscopy center in the context of their specific chronic conditions. Notably, $40 \%$ of participants reported living in rural areas. One participant said:

[...] the distance up here is a problem, because I do have urological problems [...] I do have to go to the bathroom quite often and that becomes a hardship to sit on a van $[\ldots]$ it is really a serious problem.

\section{4) Heightened concerns about potential complications}

Another theme that emerged was heightened concerns about safety and risk for potential complications mostly for individuals with cardiopulmonary issues. In at least one 
case, this led to cancelation/rescheduling and contributed to patient discouragement.

On my first colonoscopy, the doctor voided it, because I was breathing improperly. On the second, I was scheduled [....] in the operating room because I had a lack of oxygen in my blood [...] The doctor says I'm sorry I'm not doing this colonoscopy on you today [...] so I felt discouraged.

I'm on Coumadin, that's a blood thinner. So I have to be off of it for a week. If they go in there and find polyps, they're going to snip them and then there (could be) bleeding.

\section{Discussion}

This study highlights the influence of chronic conditions on motivation or ability to complete a scheduled colonoscopy in individuals with MCCs. The findings provide insight into some of the drivers and barriers to colonoscopy completion in this subgroup of individuals.

Decisions to complete the colonoscopy were based on competing medical priorities, low perceived benefit, and a prior negative experience. Individuals with MCCs reported negative prior experiences with colonoscopy because of ineffective sedation and increased postprocedure side effects (nausea). Additionally, individuals expressed heightened concerns about commonly-described barriers such as difficulty with the bowel preparation and travel. A unique barrier that emerged in this population was the concern that preparing for colonoscopy (dietary and medication changes and bowel cleansing) could potentially lead to an exacerbation of their pre-existing chronic conditions.

We also found that chronic conditions are common among individuals referred for colonoscopy (participant average of 7.4 conditions). The five most prevalent conditions were hypertension, hyperlipidemia, osteoarthritis/ degenerative joint disease, atherosclerotic heart disease, or diabetes mellitus. This is consistent with other studies examining multimorbidity. In a cohort of 897,614 nonelderly patients with $\geq 3$ conditions who received care at a VA hospital between 2009 and 2010, the most common triad of chronic conditions was diabetes, hyperlipidemia, and hypertension, which affected $24 \%$ of patients. ${ }^{9}$ Similarly, among a Medicare population, high rates of hypertension, high cholesterol, diabetes, and ischemic heart disease were among the most prevalent disease combinations. ${ }^{24}$

While many prior qualitative studies have examined factors associated with adherence to colonoscopy, no studies have primarily focused on individuals with MCCs. A recent narrative review including 56 studies summarized the published literature on patients' perceptions of the colonoscopy experience. ${ }^{25}$ The majority of studies focused on 1) perceived barriers to screening colonoscopy prior to physician recommendation, 2) perceived challenges of the colonoscopy experience after colonoscopy completion, or 3) understanding barriers unique to specific racial, ethnic, or underserved patient populations. In this review, the most consistently reported barriers to colonoscopy included inconvenience and discomfort from the laxative, anticipation of pain from the procedure, anxiety and worry about potential complications, logistical challenges with transportation, scheduling, and (for some) cost. ${ }^{25}$ Participants in our study expressed many of these same barriers and some of the logistical challenges, such as travel and laxative tolerance were perceived to be especially challenging in the context of their MCCs. We found only two studies that specifically examined patients' perceptions of barriers to colonoscopy completion in the setting of MCCs. Lukin et al surveyed African-Americans $(n=159)$ participating in a randomized clinical trial of different print educational interventions and examined the impact of chronic conditions on patients' perspectives regarding "readiness" or ability to undergo colonoscopy. ${ }^{26}$ The authors found that chronic conditions did not influence patients' readiness nor their perceived ability to undergo colonoscopy; however, they found lower rates of colonoscopy completion in individuals with "extreme" comorbidities (defined by the authors as conditions extremely likely to influence patients' ability to complete a colonoscopy, such pulmonary embolism, endocarditis, and pancreatitis). An important limitation of this study was that the majority of participants were urban, low-income, and African-American women with few chronic conditions or conditions deemed to have "minimal influence" on their ability to complete a colonoscopy $(n=71)$ such as reflux disease. Additionally, patients' perceived barriers to completing colonoscopy were not assessed. Interestingly, of the total 237 individual informed about the study, 56 refused to participate, giving reasons such as "other medical problems they were dealing with, having too many other tests, not believing it was beneficial, or being a full-time caretaker."

In another study that focused on identifying patient factors associated with colonoscopy nonattendance, the authors reported that "being too ill to attend the colonoscopy" was one of the top three reasons for nonattendance. ${ }^{27}$ In this Canadian study, the authors interviewed nonadherent individuals $(n=49)$ via telephone using open-ended questions to elicit patient reasons for nonattendance: a majority reported feeling unwell with symptoms related to either a pre-existing medical condition or an acute problem (eg, an 
upper respiratory tract infection). Ten nonattenders stated they were unable to complete their bowel preparation, felt too ill or weak from the preparation, or completed the preparation ineffectively. Unfortunately, the authors provided no additional information on the number or types of conditions.

\section{Strengths and limitations}

Our study focused on a single-center Veteran male population; studies have demonstrated higher rates of chronic disability among Veterans ${ }^{8,9}$ and gender disparities in colorectal cancer screening, with women less likely to complete screening $^{28}$ and gender-specific attitudes and barriers. ${ }^{29}$ Therefore, the barriers that we identified may not be generalizable. Additionally, we did not specifically examine the impact of MCCs on functional and cognitive status. The results that we present are a reanalysis of the focus group transcripts based on the context of examining these barriers associated with MCCs and not the primary analysis. However, this kind of additional in-depth analysis (with a more intensive focus on a particular finding or aspect than was undertaken as part of the primary work) has been shown to be a valuable form of secondary analysis of qualitative data. ${ }^{30}$ As with many qualitative studies, the results of this study should be viewed as hypothesis generating. Additional research examining barriers to colonoscopy completions, stratified by comorbid status, as well as research examining potential intervention targets for individuals with chronic medical conditions may be an important area of future research. Our choice of focus groups (as opposed to individual interviews) reflects this outlook; focus groups are an approach for identifying trends in exploratory topics.

\section{Implications for practice and research}

Our study has important clinical implications and provides insights on how to move toward a more patient-centered approach to colonoscopy. First, while not the focus of this study, ensuring the appropriateness of a colonoscopy recommendation is important. Many of the participants recognized the close tradeoff between benefits and harms in light of their pre-existing comorbidities and risk for complications. In these individuals, utilizing a shared decision-making approach and emphasizing the benefits of colonoscopy may be valuable in increasing adherence. Second, timing of colonoscopy recommendations is important as individuals may place a higher priority on dealing with competing conditions that are more acute instead of colonoscopy completion. Additionally, in circumstances where a colonoscopy is recommended for average-risk screening, pursuing alternative noninvasive forms of colorectal cancer screening may be more feasible and likely to improve adherence. Finally, because self-management is an important aspect of colonoscopy preparation, a more detailed assessment of the overall cumulative burden from chronic conditions, competing responsibilities, availability of social support, and an individual's skills and abilities may be necessary.

Research efforts aimed at developing interventions to improve adherence in individuals with MCCs may benefit from incorporating frameworks such as the cumulative complexity model that explicitly consider the impact of 1) treatment burden and illness burden imposed by chronic conditions, 2) the impact of social factors, and 3) an individual's ability to manage these conditions. ${ }^{31}$ Additional strategies to improve adherence focusing on logistical barriers should be explored such as modified (low volume) bowel preparation regimens with adjunctive antiemetic therapy or an overnight admission to the hospital for observation to help with colonoscopy preparation among individuals with impaired functional status and limited social support. However, the effectiveness and resource implications of such interventions need to be evaluated.

\section{Conclusion}

Our findings suggest that chronic conditions may generate unique barriers to colonoscopy completion. Future research should examine whether tailored interventions that include additional education and support in addressing the unique barriers related to medication, dietary changes, transportation, preparation processes, symptoms exacerbation, and complication concerns can enhance colonoscopy completion. Our findings underscore the importance of a patient-centered approach to care that includes an understanding of the factors that impact a patient's ability to prepare for and complete a colonoscopy in the context of managing other chronic conditions. . $2,32,33^{2}$

\section{Acknowledgments}

The authors would like to thank the individuals who participated in this study and Ms Lexi Charnas for her assistance with study recruitment. This material is the result of work supported with resources and the use of facilities at the Malcom Randall Veterans Affairs Medical Center, Gainesville, FL, USA, and the Minneapolis Veterans Affairs Medical Center, Minneapolis, MN, USA. This work was supported by Career Development Award 10-022 from the Department of Veterans Affairs Health Services Research and Development (S Sultan). Dr Partin is supported by a Department of Veterans Affairs Health Service Research and Development Award, RCS 10-185. The funding source had no role in the study design, collection, analysis or 
interpretation of the data, writing of the report, or the decision to submit this article for publication. The contents do not represent the views of the US Department of Veterans Affairs or the United States Government.

\section{Author contributions}

$\mathrm{SS}, \mathrm{RB}, \mathrm{BC}, \mathrm{CN}$, and SF contributed to conception and design. IMF, SS, RB, and JL contributed to analysis and interpretation of data. SS, MP, BC, RB, IMF, and JL contributed to drafting of the article. SS, MP, BC, RB, IMF, $\mathrm{JL}$, and $\mathrm{CN}$ contributed to critical revision of the article for important intellectual content. All authors contributed to final approval of the article. All authors contributed toward data analysis, drafting and critically revising the paper and agree to be accountable for all aspects of the work.

\section{Disclosure}

The authors report no conflicts of interest in this work.

\section{References}

1. Epstein RM, Street RL. The values and value of patient-centered care. Ann Fam Med. 2011;9(2):100-103.

2. Gerteis M, Edgeman-Levitan S, Daley J, Delbanco T. Through the Patient's Eyes: Understanding and Promoting Patient-Centered Care. San Francisco: Jossey-Bass; 1993.

3. Goodman RA, Posner SF, Huang ES, Parekh AK, Koh HK. Defining and measuring chronic conditions: imperatives for research, policy, program, and practice. Prev Chronic Dis. 2013;10:E66.

4. Anderson G [webpage on the Internet]. Chronic Conditions: Making the Case for Ongoing Care. Robert Wood Johnson Foundation; 2010. Available from: http://www.rwjf.org/content/rwjf/en/researchpublications/find-rwjf-research/2010/01/chronic-care.html. Accessed September 1, 2016.

5. United States Department of Health and Human Services [webpage on the Internet]. HHS Initiative on Multiple Chronic Conditions. United States Department of Health and Human Services; 2015. Available from: http://www.hhs.gov/ash/initiatives/mcc/. Accessed September 1, 2016.

6. Parekh AK, Barton MB. The challenge of multiple comorbidity for the US health care system. JAMA. 2010;303(13):1303-1304.

7. Parekh AK, Goodman RA, Gordon C, Koh HK; HHS Interagency Workgroup on Multiple Chronic Conditions. Managing multiple chronic conditions: a strategic framework for improving health outcomes and quality of life. Public Health Rep. 2011;126(4):460-471.

8. Zulman DM, Chee CP, Wagner TH, et al. Multimorbidity and healthcare utilisation among high-cost patients in the US Veterans Affairs Health Care System. BMJ Open. 2015;5(4):e007771.

9. Yoon J, Zulman D, Scott JY, Maciejewski ML. Costs associated with multimorbidity among VA patients. Med Care. 2014;52(suppl 3): S31-S36.

10. Bodenheimer T, Chen E, Bennett HD. Confronting the growing burden of chronic disease: can the U.S. health care workforce do the job? Health Aff. 2009;28(1):64-74.

11. Steinman MA, Lee SJ, Boscardin WJ, et al. Patterns of multimorbidity in elderly Veterans. J Am Geriatr Soc. 2012;60(10):1872-1880.

12. American Geriatrics Society Expert Panel on the Care of Older Adults with Multimorbidity. Patient-centered care for older adults with multiple chronic conditions: a stepwise approach from the American Geriatrics Society. J Am Geriatr Soc. 2012;60(10):1957-1968
13. Boyd CM, Fortin M. Future of multimorbidity research: how should understanding of multimorbidity inform health system design? Public Health Rev. 2010;32(2):451-474.

14. Eton DT, Ridgeway JS, Tiedje K, et al. Finalizing a measurement framework for the burden of treatment in complex patients with chronic conditions. Patient Relat Outcome Meas. 2015;6:117-126.

15. Fisher DA, Glanko J, Dudley TK, Shaheen NJ. Impact of comorbidity on colorectal cancer screening in the Veterans Health Care System. Clin Gastroenterol Hepatol. 2007;5(8):991-996.

16. Walter $\mathrm{LC}$, Lindquist $\mathrm{K}$, Nugent $\mathrm{S}$, et al. Impact of age and comorbidity on colorectal cancer screening among older Veterans. Ann Intern Med. 2009;150(7):465-473.

17. Tong A, Sainsbury P, Craig J. Consolidated criteria for reporting qualitative research (COREQ): a 32-item checklist for interviews and focus groups. Int J Qual Health Care. 2007;19(6):349-357.

18. U.S. Preventive Services Task Force [webpage on the Internet]. Final Update Summary: Colorectal Cancer: Screening. U.S. Preventive Services Task Force; 2015. Available from: https://www. uspreventiveservicestaskforce.org/Page/Document/UpdateSummaryFinal/colorectal-cancer-screening. Accessed March 3, 2017.

19. Corbin JM, Strauss A. Basics of Qualitative Research: Techniques and Procedures for Developing Grounded Theory. 4th ed. London: SAGE Publications Inc; 2014.

20. Pope C, Ziegland S, Mays N. Qualitative research in health care. Analysing qualitative data. BMJ. 2000;320(7227):114-116.

21. Charlson ME, Pompei P, Ales KL, MacKenzie CR. A new method of classifying prognostic comorbidity in longitudinal studies: development and validation. J Chronic Dis. 1987;40(5):373-383.

22. Deyo RA, Cherkin DC, Ciol MA. Adapting a clinical comorbidity index for use with ICD-9-CM administrative databases. J Clin Epidemiol. 1992;45(6):613-619.

23. Walter LC, Bertenthal D, Lindquist K, Konety BR. PSA screening among elderly men with limited life expectancies. JAMA. 2006; 296(19):2336-2342.

24. Centers for Medicare and Medicaid Services. Chronic Conditions among Medicare Beneficiaries, Chartbook. 2012 ed. Baltimore: Centers for Medicare and Medicaid Services; 2012.

25. McLachlan SA, Clements A, Austoker J. Patients' experiences and reported barriers to colonoscopy in the screening context - a systematic review of the literature. Patient Educ Couns. 2012;86(2):137-146.

26. Lukin DJ, Jandorf LH, Dhulkifl RJ, et al. Effect of comorbid conditions on adherence to colorectal cancer screening. J Cancer Educ. 2012;27(2): 269-276.

27. Chopra D, Hookey LC. Comorbid illness, bowel preparation, and logistical constraints are key reasons for outpatient colonoscopy nonattendance. Can J Gastroenterol Hepatol. 2016;2016:2179354.

28. Brawarsky P, Brooks DR, Mucci LA, Wood PA. Effect of physician recommendation and patient adherence on rates of colorectal cancer testing. Cancer Detect Prev. 2004;28(4):260-268.

29. Friedemann-Sánchez G, Griffin JM, Partin MR. Gender differences in colorectal cancer screening barriers and information needs. Health Expect. 2007;10(2):148-160.

30. Heaton J [webpage on the Internet]. Secondary Analysis of Qualitative Data. Department of Sociology, University of Surrey; 1998. Available from: http://sru.soc.surrey.ac.uk/SRU22.html. Accessed September 1, 2016.

31. Shippee ND, Shah ND, May CR, Mair FS, Montori VM. Cumulative complexity: a functional, patient-centered model of patient complexity can improve research and practice. J Clin Epidemiol. 2012;65(10): 1041-1051.

32. Institute of Medicine. Living Well with Chronic Illness: A Call for Public Health Action. Washington, DC: National Academy of Sciences; 2012.

33. Weiner SJ. Contextualizing medical decisions to individualize care. Lessons from the qualitative sciences. $J$ Gen Intern Med. 2004;19(3): $281-285$. 


\section{Supplementary material}

Table SI Primary analysis and coding tree

\begin{tabular}{|c|c|c|c|}
\hline \multicolumn{4}{|l|}{ Primary Coding Tree } \\
\hline Code & & & Definition \\
\hline \multirow[t]{8}{*}{ Poor communication } & Procedure and & & Poorly communicated instructions on the bowel prep or \\
\hline & requirements & & hospital policies (ie, having someone sign you out) \\
\hline & Results/follow-up & & $\begin{array}{l}\text { Colonoscopy results, follow-up procedures needed, etc. } \\
\text { not sufficiently communicated to patient }\end{array}$ \\
\hline & System navigation & & Complaints about not getting someone on the phone, \\
\hline & frustration & & scheduling, logistics, provider contact, etc. \\
\hline & CRC screening options & & Lack of communication on colonoscopy alternatives \\
\hline & Weak provider & & Insufficient rationale for procedure, or did not stress \\
\hline & recommendation & & importance or urgency \\
\hline Prep tolerability & & & $\begin{array}{l}\text { Difficulties with bowel preparation (fasting, bloating, } \\
\text { nausea, etc.) }\end{array}$ \\
\hline \multirow[t]{4}{*}{ Scheduling } & Non patient-centered & & Inconvenient appointment time or no/limited choice in \\
\hline & scheduling & & appointment time \\
\hline & Punitive scheduling & & Scheduling policy related to no-shows \\
\hline & Rescheduling & & Delay or difficulty rescheduling after a missed appointment \\
\hline $\begin{array}{l}\text { Lack of trust/satisfaction } \\
\text { in medical system }\end{array}$ & Negative comments on medi & al system & \\
\hline \multirow[t]{5}{*}{ Fear } & Fear of $C R C$ diagnosis & & Fear of finding out one has cancer \\
\hline & Fear of procedure & & \\
\hline & & Fear of complications & $\begin{array}{l}\text { Fear of adverse events such as perforation, anesthesia } \\
\text { problems, etc. }\end{array}$ \\
\hline & & Fear of pain & Fear of pain or discomfort from procedure \\
\hline & & Fear of ineffective sedation & Fear that sedation will or will not work \\
\hline Competing medical & & & Acute or chronic comorbidities that make prep, \\
\hline conditions & & & procedure or recovery difficult \\
\hline \multirow[t]{2}{*}{ Media } & & & Information from media (eg: patients infected with HIV at \\
\hline & & & Miami VA due to unsterilized equipment) \\
\hline Previous negative & Procedure pain & & Pain during procedure \\
\hline experience & Postprocedure side effects & & Pain or side effects after procedure \\
\hline \multirow[t]{2}{*}{ Burden to others } & & & Inconvenience to support person due to their work \\
\hline & & & schedule, travel, length of procedure, etc. \\
\hline Distance/travel & & & Issues related to traveling for the procedure \\
\hline Lack of knowledge & & & $\begin{array}{l}\text { Lack of/limited knowledge regarding procedure, risk } \\
\text { factors, CRC, etc. }\end{array}$ \\
\hline Negative peer report & & & "War Stories" - negative experiences of others \\
\hline \multirow[t]{2}{*}{ Cost } & Direct cost & & Cost of procedure or prep \\
\hline & Indirect cost & & Financial concerns related to travel, time off work, etc. \\
\hline Lack of responsible & & & Lack of person to drive and sign them in/out at the \\
\hline person & & & medical center \\
\hline Competing life events & & & Conflicts due to work or personal life \\
\hline Parking & & & Limited parking spaces \\
\hline "Hurry up and wait" & & & Frustration with the time spent between arriving at the \\
\hline & & & hospital and receiving procedure \\
\hline Modesty & & & Embarrassment \\
\hline Personal perceptions/ & & & Perception of importance, benefit, experience, outcomes \\
\hline beliefs & & & of colonoscopy \\
\hline
\end{tabular}

Abbreviations: CRC, colorectal cancer; prep, preparation; VA, Veterans Affairs.

\section{Publish your work in this journal}

Patient Preference and Adherence is an international, peer-reviewed, open access journal that focuses on the growing importance of patient preference and adherence throughout the therapeutic continuum. Patient satisfaction, acceptability, quality of life, compliance, persistence and their role in developing new therapeutic modalities and compounds to optimize clinical outcomes for existing disease states are major areas of interest for the journal. This journal has been accepted for indexing on PubMed Central. The manuscript management system is completely online and includes a very quick and fair peer-review system, which is all easy to use. Visit http://www. dovepress.com/testimonials.php to read real quotes from published authors. 